\title{
Electric field-induced translocation of single-stranded DNA through a polarized carbon nanotube membrane
}

\author{
Yinghong $\mathrm{Xie}^{\mathrm{a})}$ \\ Department of Mechanical Engineering, The University of Hong Kong, Hong Kong \\ Yong Kong \\ Max Planck Institute for Metals Research, Heisenbergstrasse 3, D-70569 Stuttgart, Germany
}

\author{
A. K. Soh \\ Department of Mechanical Engineering, The University of Hong Kong, Hong Kong \\ Huajian Gao \\ Division of Engineering, Brown University, Providence, Rhode Island 02912, USA
}

(Received 2 April 2007; accepted 22 September 2007; published online 11 December 2007)

\begin{abstract}
Molecular dynamics simulations based on a novel polarizable nanotube model were performed to study the dynamics in translocation of a single-stranded deoxyribonucleic acid oligonucleotide through a polarized carbon nanotube membrane by an applied electric field. The study revealed a nonlinear dependence of translocation velocity and an inverse quadratic dependence of translocation time on the electric field strength, as well as a threshold electric field below which the translocation process becomes impossible. The translocation rate was found to be pore-size dependent. The polarizable nanotube model developed for this study provides a useful platform for investigating the dynamics of a range of bionanosystems. (C) 2007 American Institute of Physics.
\end{abstract}

[DOI: $10.1063 / 1.2799989$ ]

\section{INTRODUCTION}

Deoxyribonucleic acid (DNA) has generated wide scientific interest due to its central role as basic hereditary material in living organisms. In the past decade, significant progresses ${ }^{1-4}$ have been made in controlling, manipulating, and characterizing the behavior of single DNA molecules in both in vitro and in vivo environments. A central problem in devising potential nanofluidic systems for rapid and accurate analysis of biomolecules involves the active translocation of biological molecules across a natural or artificial membrane. Examples include the import of particular proteins through cellular membranes and permeation of DNA or RNA molecules across nuclear pores. ${ }^{5}$ An ability to control single molecule translocation processes will have important applications in drug delivery and gene therapy technologies. ${ }^{6}$ For example, bacteria $\alpha$-hemolysin $(\alpha$-HL) channels embedded in a lipid bilayer membrane was proposed by Kasianowicz et $a l .^{7}$ for ultrafast nucleic acid detection, sequencing, and characterization; in such a device, the variation of ionic currents during biopolymers translocation was monitored and recorded for the purpose of investigating the dependence of these signals on polymer size, sequence, and composition. Subsequently, DNA molecules immersed in an electrolyte were threaded through a single $\alpha$-hemolysin pore to study the effects of the applied electric field, the length and geometry of DNA, and the temperature on translocation time and velocity; ${ }^{8}$ these have been theoretically verified by Loebl et al. $^{9}$ and Slonkina and Kolomeisky. ${ }^{10}$

\footnotetext{
a) Author to whom correspondence should be addressed. Electronic mail: xieyh@hkusua.hku.hk
}

In addition to biological membranes, inorganic solidstate pores such as synthetic $\mathrm{Si}_{3} \mathrm{~N}_{4}$ nanopores ${ }^{11,12}$ have been fabricated to conduct translocation experiments in view of their excellent stability, high tolerance for a range of temperatures and voltages, as well as good adaptability to the ambient environment. Carbon nanotubes ${ }^{13}$ (CNTs) also show great promise as nanometer-scale channels or pores for transporting water, ${ }^{14}$ proton, ${ }^{15}$ ionic flow, ${ }^{16}$ and biopolymers. ${ }^{17,18}$ Gao et al. ${ }^{17}$ reported molecular dynamics (MD) studies of spontaneously inserting single-stranded DNA (ssDNA) fragments inside carbon nanotubes and found a critical tube size below which spontaneous encapsulation becomes impossible. Yeh and Hummer ${ }^{18}$ studied the electrophoretic translocation of single-stranded RNA molecules through hexagonally packed carbon nanotube arrays upon the imposition of an external electric field; these authors pointed out that the distinct conformational flexibility and hydrophobicity of RNA molecules lead to sequence-dependent translocation.

Most existing MD simulations treat CNTs as electrically neutral molecules, without accounting for polarization due to delocalized $\pi$ electrons ${ }^{19}$ which become especially important when the CNTs are subjected to an external electric field. Neglecting the polarizable nature of CNTs can be regarded as a severe limitation of current MD simulations of CNTs. To address this limitation, we have recently developed a modified force field method ${ }^{20}$ which incorporates the electrical polarization of CNTs. In the present paper, we use this method to simulate translocation of ssDNA molecules through a polarized nanotube membrane under an applied electric field. The effects of electric field strength, the pore 

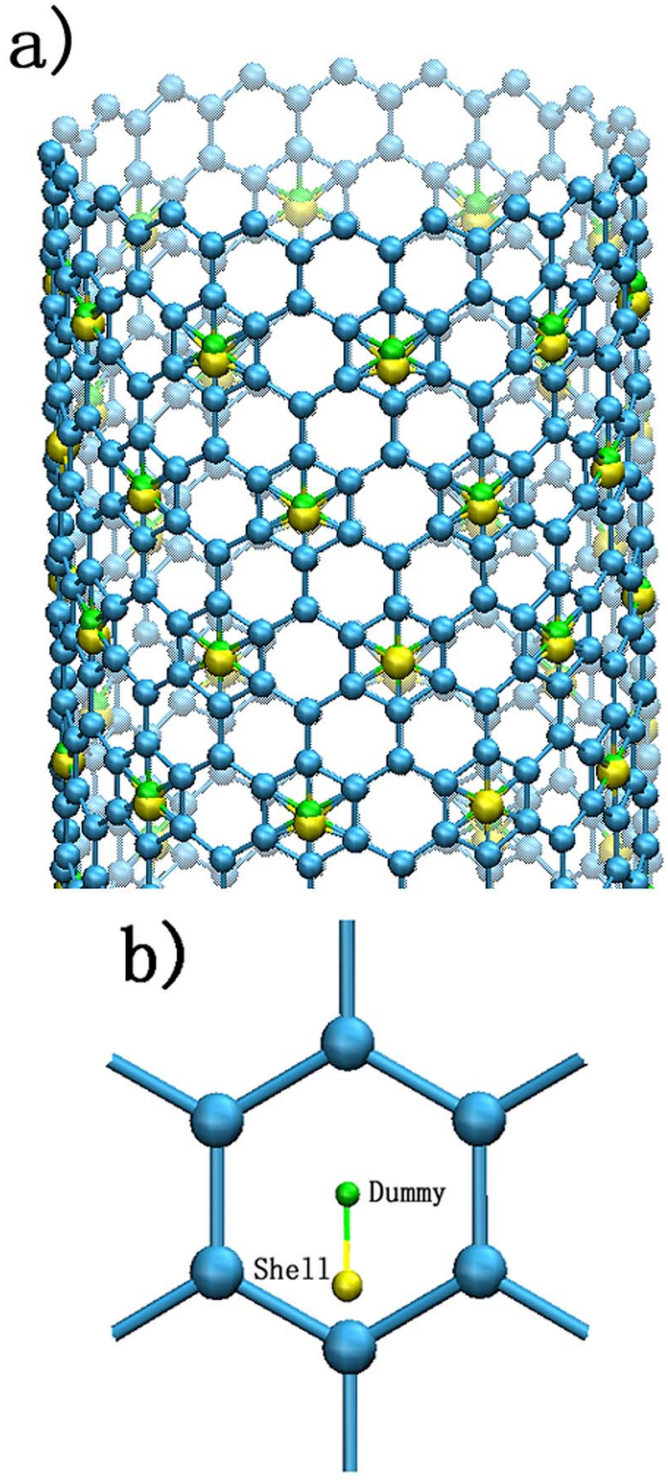

FIG. 1. (Color online) Schematic representation of the shell nanotube model. (a) Symmetrical arrangement of a polarized CNT with shell particles (yellow) and dummy atoms (green). (b) A polarized hexagonal cell with a shell particle coupled to a dummy atom via a springlike connection.

length and diameter, the size, and composition of DNA molecules on translocation dynamics will be discussed in some detail in the following sections.

\section{MODEL}

\section{A. Shell nanotube membrane model}

In our proposed shell model of polarizable carbon nanotubes, ${ }^{20}$ the electronic degrees of freedom are modeled through a network of charged shell particles whose movements reflect changes in charge distribution in the nanotube in response to an external electric field. The shell particles are periodically distributed within the nanotube [Fig. 1(a)]; each shell particle is located near the center of a hexagonal cell and surrounded by six carbon atoms positioned at the vertices of the cell [Fig. 1(b)]. The carbon atoms in any hexagonal cell containing a shell particle are assumed to be positively charged while the rest of the carbon atoms remain

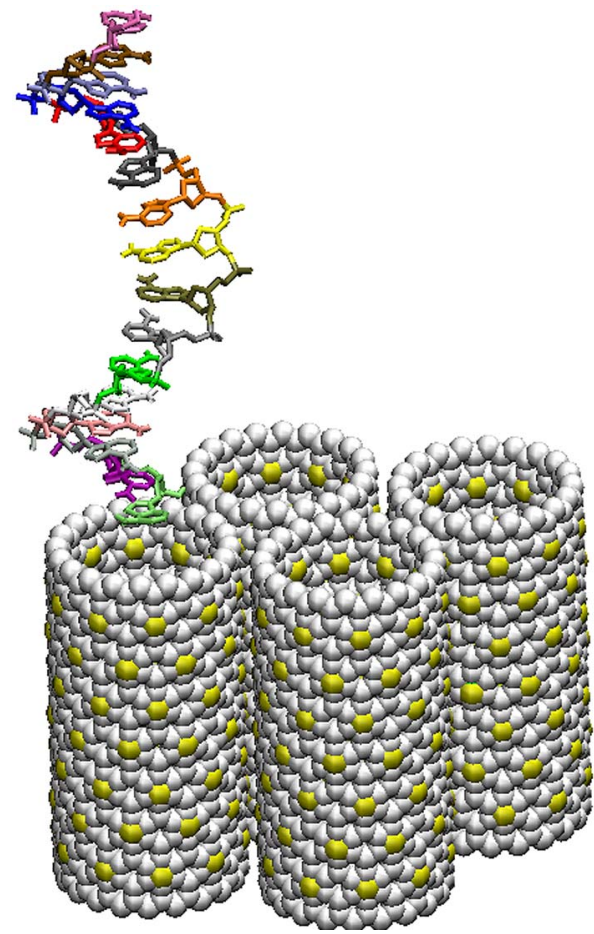

FIG. 2. (Color online) A designed CNT membrane-DNA complex where the DNA strand is aligned with one of the four packed nanotubes.

uncharged. To keep electrical neutrality, the shell particle is assigned a negative charge with magnitude equal to the total charge of the six surrounding carbon atoms. At the level of a hexagonal cell, the polarization of CNT is accomplished through the movement of the shell particle relative to a dummy atom located at the center of the cell. The shell particle is assumed to be coupled to the massless and uncharged dummy atom via a harmonic spring with force constant $k_{i}$ $=q_{s}^{2} / 4 \pi \varepsilon_{0} \alpha_{z z}$ and a zero equilibrium distance, where $\varepsilon_{0}, q_{s}$, and $\alpha_{z z}$ denote, respectively, the vacuum dielectric constant, the effective charge of the shell particle, and the axial polarizability of a single cell.

Only the axial polarizability $\alpha_{z z}$ is considered in the present study because the axial polarizability, whether for a CNT or for individual carbon atoms, is usually an order of magnitude larger than the transverse polarizability, ${ }^{21,22}$ especially in the cases when the net dipole moments are aligned primarily along the axial direction of the tube in response to an electric field applied parallel to the axial direction. To avoid complications in divergence of polarizability and dielectric constants for metallic nanotube, ${ }^{21}$ we focus our attention on semiconducting single-walled nanotubes (SWNTs).

\section{B. Simulations}

The CNT-DNA system (Fig. 2) is composed of a polarized nanotube membrane and a homogeneous singlestranded DNA fragment with water as an explicit solvent and additional sodium ions for electrically neutralizing the whole system. In modeling a polarizable nanotube membrane, four zigzag nanotubes are arranged in a hexagonal pattern in the $x-y$ plane with their tube axes aligned in the $z$ direction; the 
intertube spacing is taken to be $0.34 \mathrm{~nm} .{ }^{14}$ The DNA strand is initially aligned with the axis of one of the four assembled nanotubes and situated near the entrance of the tube such that the nearest axial distance between the DNA and CNT atoms is $0.2 \mathrm{~nm}$. In most cases, the ssDNA molecules are driven electrophoretically inside a zigzag $(20,0)$ nanotube of diameter of $1.56 \mathrm{~nm}$ to ensure a simple one-dimensional translocation. ${ }^{9,23}$ The nanotube membrane-DNA complex with CNT atoms fixed at their original positions is placed at the center of a periodic rhombic-shaped box; ${ }^{20}$ the length of the box edge in the $x-y$ plane is set to be $\sim 3.9 \mathrm{~nm}$ with interior angles equal to $60^{\circ}$ or $120^{\circ}$; the box dimension in the $z$ direction is chosen according to the length of the ssDNA molecule. There are totally around 20 000-43 000 atoms involved in the simulation systems, which is primarily determined by the length/type of DNA oligonucleotide and the size of CNTs. The CNT atom involved in polarization is assigned a charge of $q_{c}=0.05 e$, and the shell particle is given a charge of $q_{s}=-0.3 e$. The magnitude of the axial polarizability per hexagonal cell can be acquired through the methods described by Benedict et al. ${ }^{21}$ For zigzag $(20,0)$ SWNTs, this value is calculated to be $0.129 \mathrm{~nm}^{3}$.

We have implemented the modified force field of polarizable nanotube membrane ${ }^{20}$ in the MD simulation package GROMACS, ${ }^{24}$ with water solvent described by the SPC216 (Ref. 25) model. The long-range electrostatic interactions are evaluated by the particle-mesh Ewald method. ${ }^{26}$ The LINCS algorithm $^{27}$ is employed to integrate Newton's equations of motion with a time step of 1 fs. The simulations are first equilibrated for $100 \mathrm{ps}$ in the $N P T$ ensemble, in order to ensure a constant volume for the later step. Continuously, a typical NVT ensemble is implemented once the external electric field is applied, in which a low temperature of $T=1 \mathrm{~K}$ with time constant of $1 \mathrm{fs}$ for coupling is assigned to the shell-dummy pairs, while the values of $T=300 \mathrm{~K}$ and $0.1 \mathrm{ps}$ are applied to other atoms. A strong enough electric field (at least $0.3 \mathrm{~V} / \mathrm{nm}$ ) is applied along the axial direction of nanotubes to induce rapid translocation within the nanosecond time scale. $^{18}$

\section{RESULTS AND DISCUSSION}

\section{A. Verification of shell CNT membrane model}

The feasibility of modeling polarizable nanotubes is quantitatively tested in this section. We have performed a set of simulations to illustrate the electronic behavior of the proposed shell nanotube membrane model in response to a direct current electric field. The relative motion between the negatively charged shell particles and the positively charged carbon atoms is used as an effective measure of charge redistribution when the initially neutral nanotubes are polarized by an external field, which could be interpreted as the realization of electrical polarization of CNTs. To test the dielectric response of the shell CNTs model, an electric field with prescribed strength $(0.6 \mathrm{~V} / \mathrm{nm})$ is applied along the axes of the tubes. Figure 3 shows that this field causes the shell particles to oscillate with an average magnitude of $0.12 \mathrm{~nm}$ around their corresponding dummy atoms, which is, however, one-third lower than the theoretical estimate $\left|r_{i}\right|$

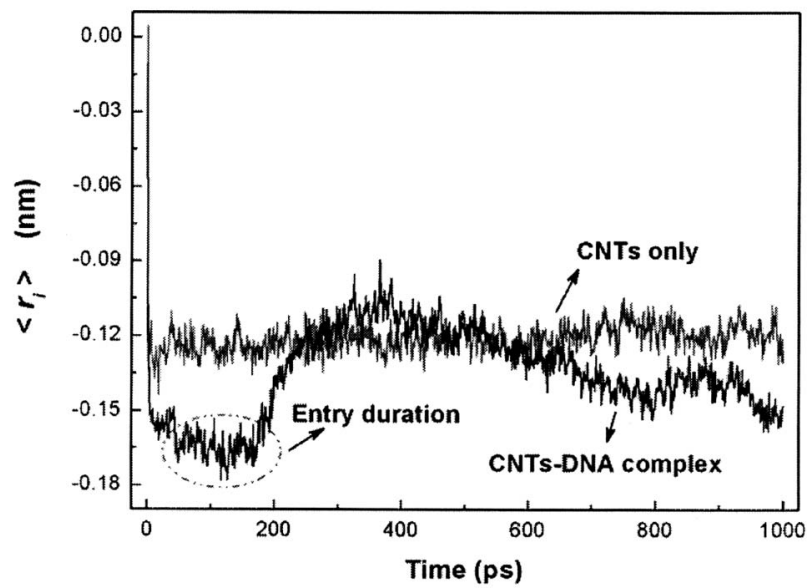

FIG. 3. Motion of shell particles in a pure CNT membrane system and a CNT-DNA complex system under an applied electric field $E=0.6 \mathrm{~V} / \mathrm{nm}$.

$=4 \pi \varepsilon_{0} \alpha_{z z}|E| /\left|q_{s}\right| \approx 0.18 \mathrm{~nm}$. This discrepancy can be attributed to the insufficient number of water molecules in the simulation box. Previous investigation of the effect of waterfilled simulation box ${ }^{28}$ has shown that the solute (e.g., a CNT membrane) should be dilated with a layer of water shell of at least half the cutoff distance to ensure reasonable accuracy. In the present model, a strict intertube spacing of $0.34 \mathrm{~nm}$ prevents us from filling sufficient water molecules between neighboring tubes. More discussions can be found in our previous paper. $^{20}$

The motion of shell particles in the CNT membraneDNA complex system is also plotted in Fig. 3. The difference from that of pure CNT membrane is caused by the Coulomb and Lennard-Jones (LJ) interactions between the negatively charged ssDNA molecules and shell particles, which is especially pronounced at the entry duration.

\section{B. Nonpolarized versus polarized CNT membrane for translocation}

In order to illustrate the effects of nanotube polarization on the electric field-driven translocation process of ssDNA, two simulations are carried out on a system, which consist of a homogeneous nucleotides of 16 adenine base (A16) and a 3.3 -nm-thick zigzag $(20,0)$ membrane that is polarized in one simulation and nonpolarized in another, and is subjected to an electric field $E=0.6 \mathrm{~V} / \mathrm{nm}$. The trajectories of translocation of the center of mass (c.o.m.) of DNA oligonucleotide relative to that of the nanotube are displayed in Fig. 4.

Generally, the implementation of nanotube polarization does slightly slow down the translocation rate of ssDNA. To explore the reason for such slow down, the distribution of electrostatic potential across the membrane is analyzed, as shown in the inset of Fig. 4. In our simulations, the external field is applied across the whole system along the $z$ direction, and the electrostatic potential generated in the bulk water is significantly lower than that inside the CNT membrane; this also plays an important role in capturing the ssDNA molecule inside the pore. The different electrostatic potential drop inside a polarized or a nonpolarized nanopore causes the different translocation velocities of DNA oligonucleotide through nanopores. One possibility is that the external elec- 


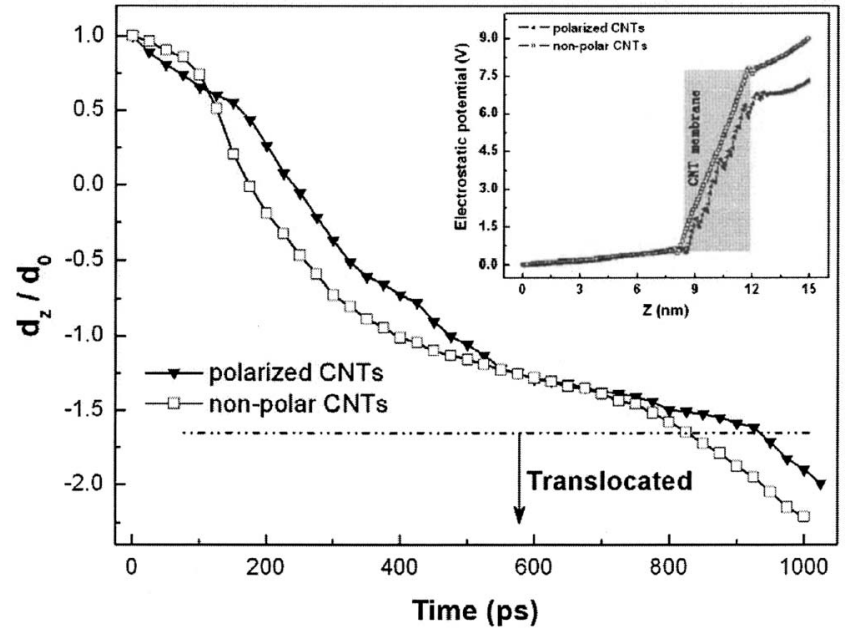

FIG. 4. The trajectories of the center of mass (c.o.m.) of A16 during translocation through a nonpolarized (squares) and a polarized nanotube membrane (triangles) under an electric field $E=0.6 \mathrm{~V} / \mathrm{nm}$. The inset presents the electrostatic potential distribution within both nanopores (over $1.5 \mathrm{~ns}$ duration of simulations).

tric field applied across the polarized nanopore gave rise to the movement of shell particles resulting in the formation of an opposite electric field pointing from the positively charged CNT atoms to negatively charged shell particles, which reduces the overall electric field across the nanopore and, thus, slightly encumbers the translocation event of ssDNA.

\section{Electric field dependence of translocation}

The selected CNT membrane-DNA complex consists of a homogeneous ssDNA strand of A16 and a 3.3-nm-thick zigzag $(20,0)$ membrane. Figure 5(a) displays the (c.o.m.) trajectories of A16 subjected to an external field with strengths $0.3,0.4$, and $0.6 \mathrm{~V} / \mathrm{nm}$. For clarity, a typical event for A16 translocation through nanopore under an electric field of $0.4 \mathrm{~V} / \mathrm{nm}$ is visualized in Fig. 5(b) by the program $\mathrm{VMD},{ }^{29}$ which shows an approximately one-dimensional permeation behavior of ssDNA through nanopore due to comparable size of A16 and zigzag $(20,0)$ nanotube, and reflects a slow release of ssDNA from the exit side of nanopore owing to the adhesion of the last several bases to the interior surface of nanopores. Limited to our simulations of several nanosecond time scale, the weaker field strength $0.3 \mathrm{~V} / \mathrm{nm}$ is seen to be inadequate to overcome the energetic barrier that tends to prevent ssDNA from exiting the nanotube. This observation is also similar to the conclusions of Aksimentiev et $a l^{30}$ and Yeh and Hummer, ${ }^{18}$ in which they found that hydrophobic attractions between nanopores and polymers significantly delayed the release of DNA or RNA from the nanopore at small applied electric field. Successful translocation is observed when the field strength is increased to above $0.4 \mathrm{~V} / \mathrm{nm}$, suggesting that there exists a threshold electric field below which no translocation can be achieved on the simulation time scale. According to the plots in Fig. 5(a), this threshold field strength should be between 0.3 and $0.4 \mathrm{~V} / \mathrm{nm}$. Our results are comparable to the MD studies ${ }^{11,31}$ on the voltage driven translocation of a double-stranded
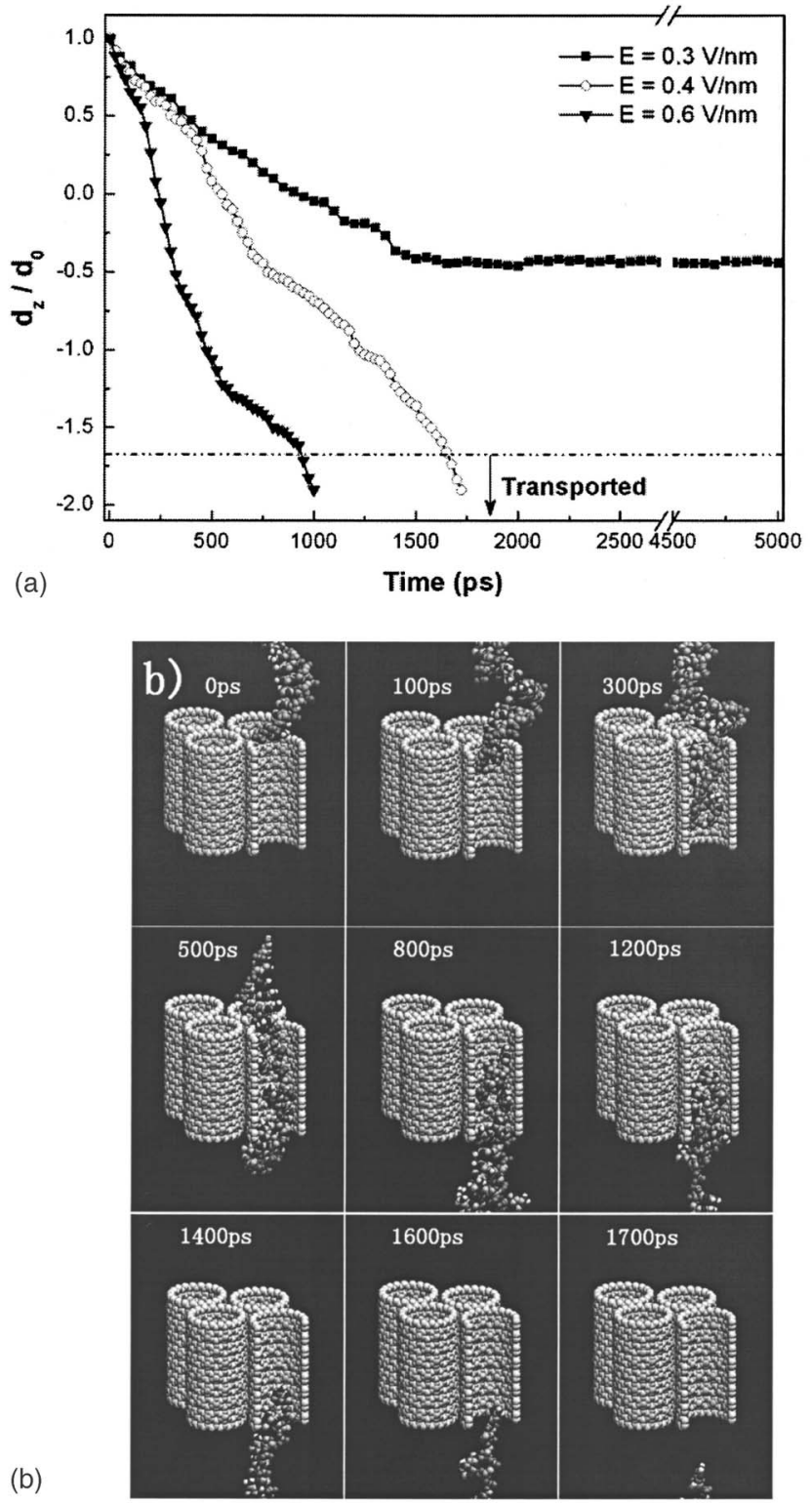

FIG. 5. (a) The (c.o.m.) positions of DNA oligonucleotide under different field strengths: $0.3 \mathrm{~V} / \mathrm{nm}$ (squares), $0.4 \mathrm{~V} / \mathrm{nm}$ (circles), and $0.6 \mathrm{~V} / \mathrm{nm}$ (triangles). (b) Snapshots of A16 conformations during its translocation through the centrally hollow cavity of CNT under an electric field $E=0.4 \mathrm{~V} / \mathrm{nm}$.

DNA (dsDNA) through a 10 -nm-thick $\mathrm{Si}_{3} \mathrm{~N}_{4}$ nanopore, in which a threshold voltage of $>2.6 \mathrm{~V}$ was found to ensure this dsDNA molecule to permeate a $1.0 \mathrm{~nm}$ radius pore within a few tens of nanoseconds.

A critical translocation force can be calculated as $F^{*}$ $=q Z \Delta V / a,{ }^{32}$ where $F^{*}$ is the electrical force acting on the ssDNA strand inside the nanopore, $q Z$ denotes the effective charge per nucleotide, $a=0.4 \mathrm{~nm}$ is the base-to-base separation, and $\Delta V$ refers to the electrostatic potential across the nanopore. The choice of $Z(0<Z<1)$ is determined by the extent of screening of the ssDNA molecule in water: $Z=1$ means that the screening effect is negligible. A threshold field of $0.35 \mathrm{~V} / \mathrm{nm}$ corresponds to a voltage drop of $5.25 \mathrm{~V}$ across the entire simulation box of $15 \mathrm{~nm}$ in length in the $z$ direction, and $\Delta V \approx 3.5 \mathrm{~V}$ over the length of the CNT nanopore. Under these assumptions, the electrical force is calcu- 


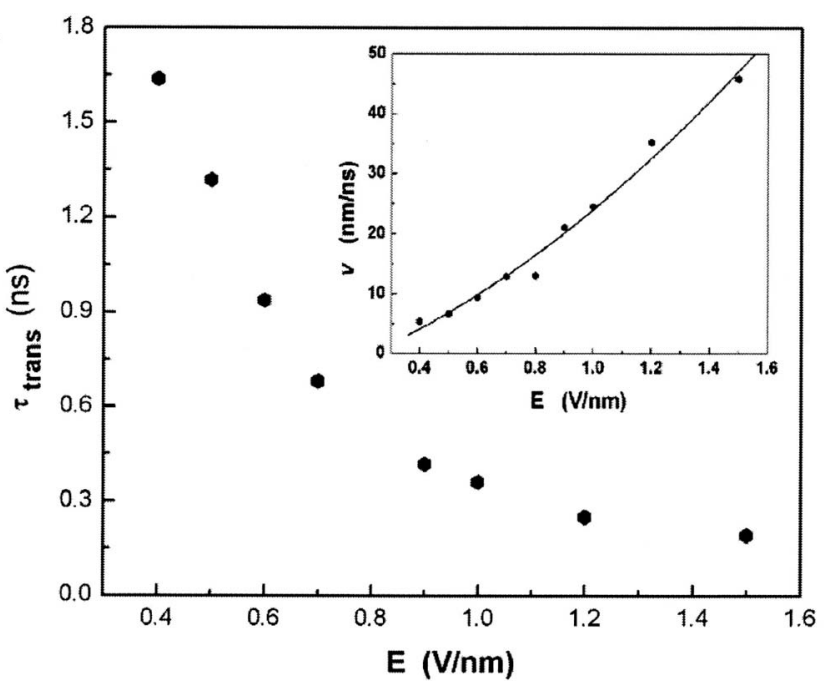

FIG. 6. Effects of electric field strength on translocation time and translocation velocity.

lated to be $\sim 1400 \mathrm{pN}$ without considering the screening effect, which is an upper limit of the critical driving force. On the other hand, a charge reduction of $75 \%$ has been experimentally reported in translocation of ssDNA by Keyser et al. ${ }^{32}$ which indicates a maximum critical force of around $350 \mathrm{pN}$ to transport 16 adenine nucleotides through the CNT membrane within the simulated duration of several nanoseconds.

We have also studied the influence of the field strength on translocation time $\tau_{\text {trans }}$ and translocation velocity, within the field range of $0.4-1.5 \mathrm{~V} / \mathrm{nm}$. Figure 6 presents an inversed quadratic relationship between the translocation time $\tau_{\text {trans }}$ and the external field strength, which, except for the difference in time scale, is in good agreement with both the experimental results of Kasianowicz et al. ${ }^{7}$ and Monte Carlo simulations of Loebl et al. ${ }^{9}$ Besides, such a relationship is comparable to Yeh and Hummer's investigations ${ }^{18}$ on the dependence of mean entry/exit time on the electric field; their MD simulations showed that the increase of the electric field, from 0.3 to $0.5 \mathrm{~V} / \mathrm{nm}$, might decrease the translocation durations of both A6 and U6 RNA fragment through nanopore. In this paper, the translocation event is defined as the duration from the entry of the first atom of ssDNA into one side of the CNT nanopore to the last atom leaving the other end of the nanopore. Accordingly, the translocation speed of A16 can be approximately estimated ${ }^{10}$ as $v=\left(l_{\mathrm{DNA}}+l_{\mathrm{CNT}}\right) / \tau_{\text {trans. }}$. A quadratic dependence of translocation velocity on the applied field is shown in the inset of Fig. 6 , which tallies with the experimental observation made by Meller et al. ${ }^{8}$

\section{Energetics of CNT-DNA interaction and the effect of pore size}

To explore the intrinsic mechanisms of ssDNA translocation process, the interactions between the CNT membrane and the ssDNA molecule are studied in this section. The electrostatic and van der Waals nonbonded potential energies
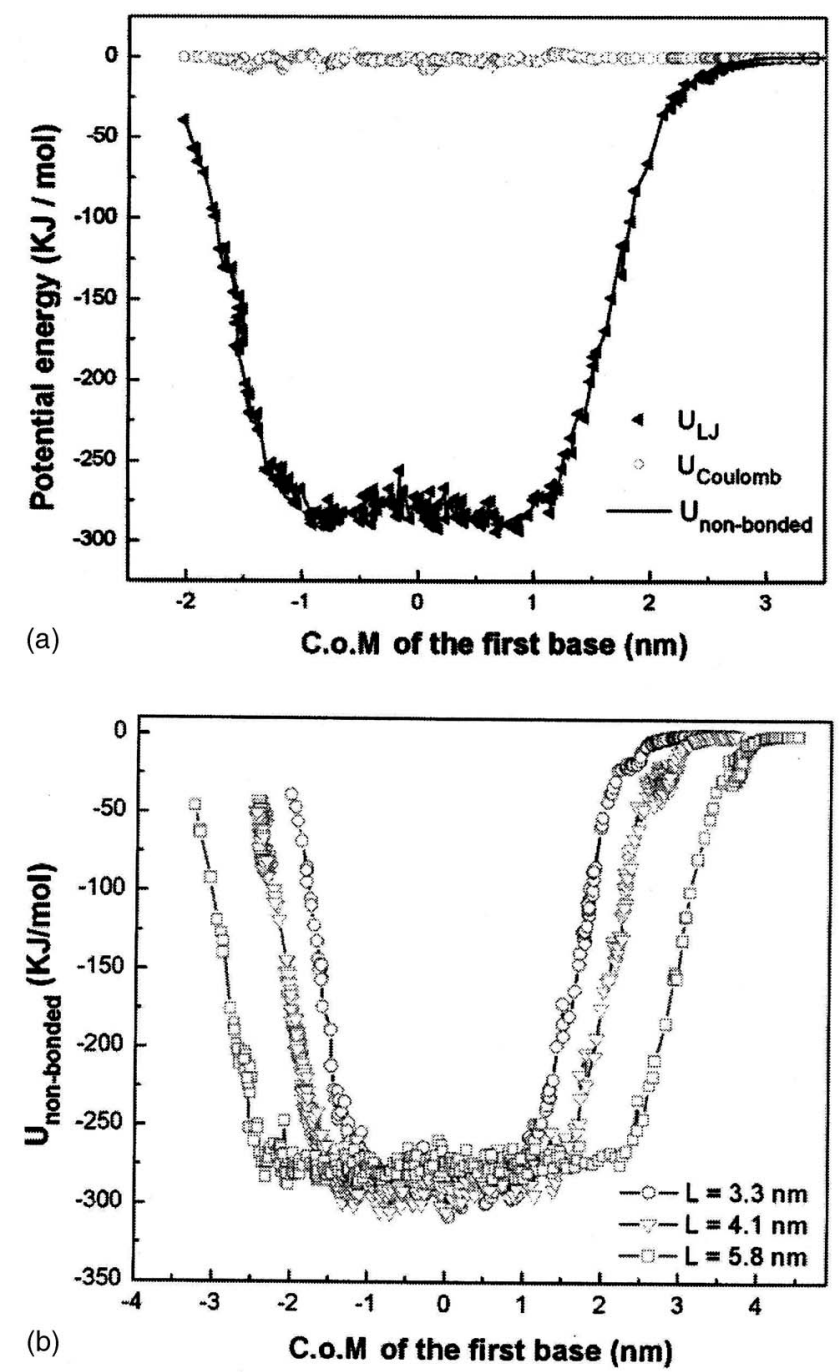

FIG. 7. (a) The electrostatic (circles), van der Waals (triangles), and nonbonded (line) interactions between the polarized nanopore and the first base of DNA oligonucleotide vs the (c.o.m.) position of the first base. (b) The nonbonded potential energy between nanopores and the first base of DNA oligonucleotide, during its translocation through nanopores with lengths of $3.3 \mathrm{~nm}$ (circles), $4.1 \mathrm{~nm}$ (triangles), and $5.8 \mathrm{~nm}$ (squares).

between the first base of 16 adenine ssDNA and the nanotube membrane of $3.3 \mathrm{~nm}$ in length and $1.56 \mathrm{~nm}$ in diameter are plotted in Fig. 7(a). The Coulomb interactions between the first base of the charged ssDNA molecule and CNT atoms appear to be negligible in comparison with the van der Waals interactions. Figure 7(a) shows that both the nonbonded and van der Waals potential are almost symmetric with respect to the middle of the nanotubes with a flat potential well of $\sim 275 \mathrm{~kJ} / \mathrm{mol}$. It is important to note that, apart from the contribution of the external electric field in driving the translocation process, the attractive van der Waals potential also plays a significant role in accelerating the encapsulation of ssDNA and delaying its exit from the nanopore. The change of nonbonded energy can be characterized according to three stages of translocation process: Entry into, moving inside, and escaping of the ssDNA from the nanopore. During the insertion of ssDNA, the nonbonded energy decreases rapidly and then becomes stabilized during the sliding of ssDNA inside the nanopore. From a thermodynamics point of view, 
the evolution of the system tends to minimize its free energy. ${ }^{33}$ During the exit stage, a sufficiently strong electric field is necessary to assist the ssDNA to overcome the energy barrier in order to escape from the nanopore. This tendency of van der Waals interactions is qualitatively similar to that of the previous investigation ${ }^{34}$ on the electric field-driven transportation of water molecules through nonpolarized nanotubes. We have conducted two additional simulations with longer nanotubes with the same diameter but 4.1 and $5.8 \mathrm{~nm}$ in length. Figure 7(b) shows that longer nanotubes produced a wider flat bottom for the potential well in the middle of the nanotube without altering the depth of the potential well.

To investigate the effect of pore size on the translocation of ssDNA, we have considered nanotubes of different diameters. In particular, zigzag $(20,0)$ and $(32,0)$ CNT membranes subjected to an electric field $(0.5 \mathrm{~V} / \mathrm{nm})$ are employed in simulations of the ssDNA translocation. The positions of the (c.o.m.) of ssDNA molecule in Fig. 8(a) show that the translocation of ssDNA in a pore of larger diameter is faster. As the primary contributor for the change of free energy in the system, the nonbonded interactions among DNA oligonucleotide, CNT membrane, and water molecules are evaluated to compare the dynamics of ssDNA molecule in pores of different diameters. Figure 8(b) shows that the nonbonded interactions between DNA oligonucleotide and polarized CNTs resulted in a much deeper potential well in the tube of smaller diameter at the insertion duration due to the shorter distance between the tube and DNA atoms. On the other hand, the occupancy of DNA strand in the pore inevitably leads to the repelling of some water molecules from the cavity of nanopore, which generates a resistance for DNA encapsulation; ${ }^{17}$ the repelling of water molecules during the insertion of ssDNA results in an increase of free energy, as plotted in Fig. 8(c). The larger decrease of nonbonded CNT-DNA interactions during the insertion of DNA into a pore of smaller diameter is overcompensated by the increasing resistance to DNA oligonucleotide arising from the water molecules being repelled. This leads to a relatively faster encapsulation process in the pore of larger diameter. For the whole translocation process, the reduction in total potential energy in the larger pore $(\sim 400 \mathrm{~kJ} / \mathrm{mol})$ is higher than that in the smaller pore $(\sim 200 \mathrm{~kJ} / \mathrm{mol})$, as shown in Fig. 8(d). This further demonstrates that the larger pore facilitates an easier translocation event.

\section{CONCLUSION}

Molecular dynamics simulations have been performed to investigate the electric field-driven translocation of singlestranded DNA molecules through polarized CNT membranes. Within the limited time scale of our simulations, a critical field strength, below which no translocation event can occur, is identified. Moreover, an inverse quadratic dependence of translocation time and a nonlinear dependence of translocation speed on the field strength have been obtained. The translocation process is found to be tube-size dependent, with larger nanopores facilitating faster translo-
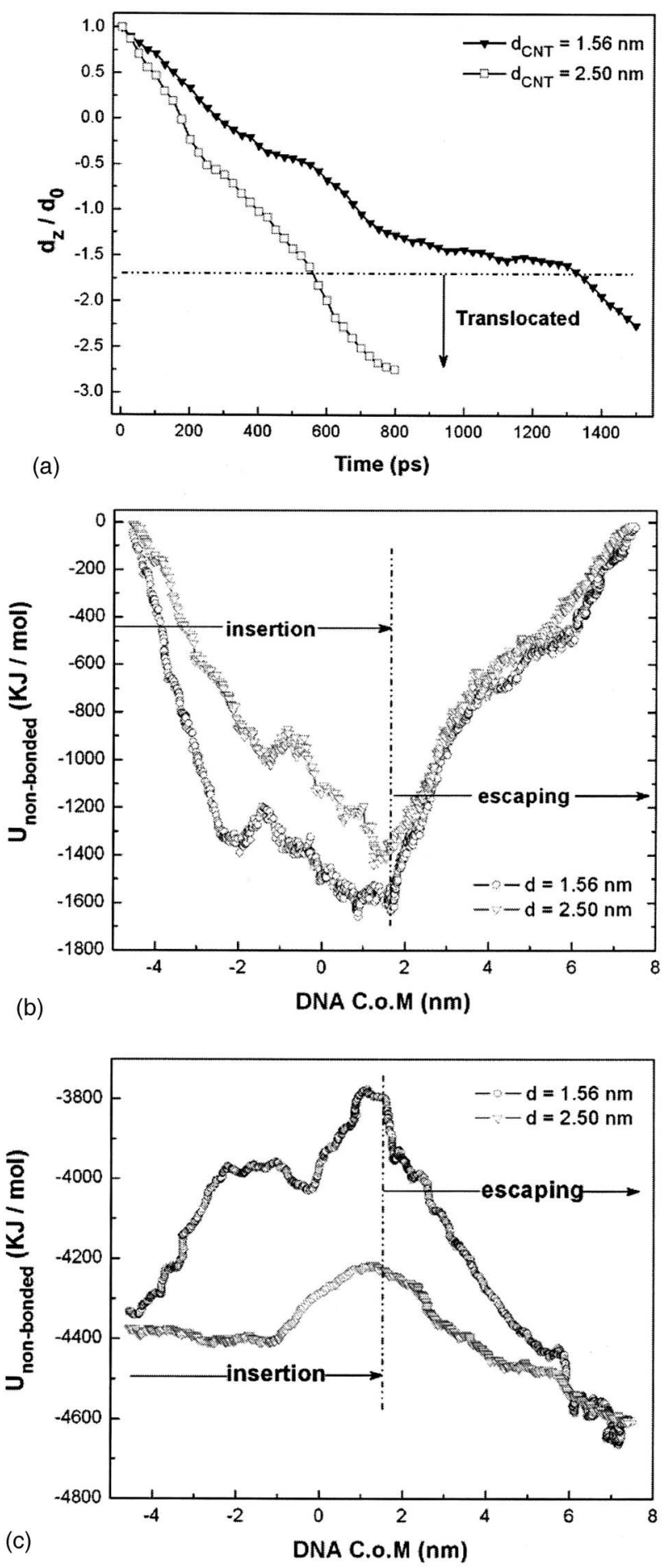

FIG. 8. (a) The (c.o.m.) trajectories of DNA nucleotides during translocation through nanopores with diameters of 1.56 and $2.50 \mathrm{~nm}$ under an electric field $E=0.5 \mathrm{~V} / \mathrm{nm}$. (b) The nonbonded potential energy between the entire DNA strand and the nanopore. (c) The nonbonded potential energy between solvent and DNA strand. (d) The reduction of total potential energy in the system vs the c.m. position of DNA.

cation. Apart from the contribution from the electric field, the nonbonded interactions also play a significant role in the ssDNA translocation. The conformational dynamics of ssDNA molecules inside a nanopore is an important factor to determine the affinity of ssDNA to the wall of nanotubes and the rate of translocation. 


\section{ACKNOWLEDGMENTS}

Support from the Research Grants Council of the Hong Kong Special Administrative Region, China (Project No. HKU 7195/04B) and the University Research Grant of the University of Hong Kong is acknowledged.

${ }^{1}$ E. L. Florin, V. T. Moy, and H. E. Gaub, Science 264, 415 (1994).

${ }^{2}$ M. L. Bennink, S. H. Leuba, G. H. Leno, J. Zlatanova, and B. G. de Grooth, Nat. Struct. Biol. 8, 606 (2001).

${ }^{3}$ D. E. Smith, H. P. Babcock, and S. Chu, Science 283, 1724 (1999).

${ }^{4}$ N. Kaji, M. Ueda, and Y. Baba, Biophys. J. 82, 335 (2002).

${ }^{5}$ B. Alberts, A. Johnson, J. Lewis, M. Raff, K. Roberts, and P. Walter, Molecular Biology of the Cell (Garland, New York, 1994).

${ }^{6}$ D. C. Chang, Guide to Electroporation and Electrofusion (Academic, New York, 1992).

${ }^{7}$ J. J. Kasianowicz, E. Brandin, D. Branton, and D. Deamer, Proc. Natl. Acad. Sci. U.S.A. 93, 13770 (1996).

${ }^{8}$ A. Meller, L. Nivon, and D. Branton, Phys. Rev. Lett. 86, 3435 (2001).

${ }^{9}$ H. C. Loebl, R. Randel, S. P. Goodwin, and C. C. Matthai, Phys. Rev. E 67, 041913 (2003).

${ }^{10}$ E. Slonkina and A. B. Kolomeisky, J. Chem. Phys. 118, 7112 (2003)

${ }^{11}$ J. B. Heng, A. Aksimentiev, C. Ho, P. Marks, Y. V. Grinkova, S. Sligar, K. Schulten, and G. Timp, Nano Lett. 5, 1883 (2005).

${ }^{12}$ A. J. Storm, C. Storm, J. Chen, H. Zandbergen, J. F. Joanny, and C. Dekker, Nano Lett. 5, 1193 (2005).

${ }^{13}$ M. S. Dresselhaus, G. Dresselhaus, and P. Avouris, Top. Appl. Phys. 80, 287 (2001).

${ }^{14}$ A. Kalra, S. Garde, and G. Hummer, Proc. Natl. Acad. Sci. U.S.A. 100 10175 (2003).

${ }^{15}$ D. J. Mann and M. D. Halls, Phys. Rev. Lett. 90, 195503 (2003).

${ }^{16}$ S. Joseph, R. J. Mashl, E. Jakobsson, and N. R. Aluru, Nano Lett. 3, 1399 (2003)
${ }^{17}$ H. Gao, Y. Kong, D. Cui, and C. S. Ozkan, Nano Lett. 3, 471 (2003).

${ }^{18}$ I. C. Yeh and G. Hummer, Proc. Natl. Acad. Sci. U.S.A. 101, 12177 (2004).

${ }^{19}$ M. S. Dresselhaus, G. Dresselhaus, and P. C. Eklund, Science of Fullerenes and Carbon Nanotubes (Academic, San Diego, CA, 1996).

${ }^{20}$ Y. H. Xie, Y. Kong, H. Gao, and A. K. Soh, "Molecular dynamics simulation of polarizable carbon nanotubes," Comput. Mater. Sci. 40, 460 (2007).

${ }^{21}$ L. X. Benedict, S. G. Louie, and M. L. Cohen, Phys. Rev. B 52, 8541 (1995).

${ }^{22}$ R. Langlet, M. Devel, and Ph. Lambin, Carbon 44, 2883 (2006).

${ }^{23}$ A. Meller, J. Phys.: Condens. Matter 15, R581 (2003).

${ }^{24}$ D. van der Spoel, E. Lindahl, B. Hess, A. R. van Buuren, E. Apol, P. J. Meulenhoff, D. P. Tieleman, A. L. T. M. Sijbers, K. A. Feenstra, R. van Drunen, and H. J. C. Berendsen, Gromacs User Manual University of Groningen, Nijenborgh, 4, 9747, AG Groningen, The Netherlands (2002).

${ }^{25}$ O. Teleman, B. Jonsson, and S. Engstrom, Mol. Phys. 60, 193 (1987).

${ }^{26}$ T. Darden, D. York, and L. Pedersen, J. Chem. Phys. 98, 10089 (1993).

${ }^{27}$ B. Hess, H. Bekker, H. J. C. Berendsenk, and J. G. E. M. Fraaije, J. Comput. Chem. 18, 1463 (1997).

${ }^{28}$ H. Bekker, J. P. van den Berg, and T. A. Wassenaar, J. Comput. Chem. 25, 1037 (2004).

${ }^{29}$ W. Humphrey, A. Dalke, and K. Schulten, J. Mol. Graphics 14, 33 (1996).

${ }^{30}$ A. Aksimentiev, J. B. Heng, G. Timp, and K. Schulten, Biophys. J. 87, 2086 (2004)

${ }^{31}$ J. B. Heng, A. Aksimentiev, C. Ho, P. Marks, Y. V. Grinkova, S. Sligar, K. Schulten, and G. Timp, Biophys. J. 90, 1098 (2006).

${ }^{32}$ U. Keyser, B. N. Koeleman, S. V. Dorp, D. Krapf, R. M. M. Smeets, S. G. Lemay, N. H. Dekker, and C. Dekker, Nat. Phys. 2, 473 (2006).

${ }^{33}$ R. S. Conroy and C. Danilowicz, Contemp. Phys. 45, 277 (2004).

${ }^{34}$ D. Lu, Y. Li, U. Ravaioli, and K. Schulten, J. Phys. Chem. B 109, 11461 (2005). 